\section{Mother's Exposure to Electromagnetic Fields before and during Pregnancy is Associated with Risk of Speech Problems in Offspring}

\author{
Zarei S. ${ }^{1}$, Vahab M.,2,3, Oryadi-Zanjani M. M.,2,3, Alighanbari \\ N. ${ }^{4}$, Mortazavi S. M J.5,6*
}

\begin{abstract}
Background: Rapid advances in technology, especially in the field of telecommunication, have led to extraordinary levels of mothers' exposures to radiofrequency electromagnetic fields (RF-EMFs) prior to or during pregnancy.

Objective: The main goal of this study was to answer this question whether exposure of women to common sources of RF-EMFs either prior to or during pregnancy is related to speech problems in the offspring.
\end{abstract}

Materials and Methods: In this study, mothers of 110 three-to-seven-yearold children with speech problems and 75 healthy children (control group) were interviewed. These mothers were asked whether they had exposure to different sources of EMFs such as mobile phones, mobile base stations, Wi-Fi, cordless phones, laptops and power lines. Chi square test was used to analyze the differences observed between the control and exposed groups.

Results: Statistically significant associations were found between the use of cordless phone and offspring speech problems for both before pregnancy and during pregnancy maternal exposures $(\mathrm{P}=0.005$ and $\mathrm{P}=0.014$, respectively). However, due to high rate of mobile phone use in both groups, this study failed to show any link between mobile phone use and speech problems in offspring. Furthermore, significant associations were observed between living in the vicinity of power lines and speech problems again for both before pregnancy and during pregnancy maternal exposures $(\mathrm{P}=0.003$ and $\mathrm{P}=0.002$, respectively). However, exposure to other sources of nonionizing radiation was not linked to speech problems. Moreover, exposure to ionizing radiation (e.g. radiography before and during pregnancy) was not associated with the occurrence of speech problems.

Conclusion: Although this study has some limitations, it leads us to this conclusion that higher-than-ever levels of maternal exposure to electromagnetic fields could be linked to offspring speech problems.

\section{Keywords}

Speech Problem, Exposure, Pregnancy, lonizing radiation, Non-ionizing Radiation, Electromagnetic Fields

\section{Introduction}

he development of speech in children varies greatly. A delay in speech may be caused by a wide variety of factors such as

L hearing loss, speech or language disorders. Problem in making speech sounds correctly is common in children with speech disorders. They also may have problems in understanding what other people say
${ }^{1}$ Department of Speech

Therapy, School of Reha-

bilitation Sciences, Shiraz

University of Medical Sci-

ences, Shiraz, Iran

${ }^{2}$ Rehabilitation Sciences

Research Center, Shiraz

University of Medical Sci-

ences, Shiraz, Iran

${ }^{3}$ Network of Childhood

Speech and Language

Disorders (NCSLD), Uni-

versal Scientific Education

and Research Network

(USERN), Tehran, Iran

${ }^{4}$ Occupational Health

Engineering Department,

School of Public Health,

Shiraz University of Medical

Sciences, Shiraz, Iran

${ }^{5}$ Medical Physics and Medi-

cal Engineering Depart-

ment, School of Medicine,

Shiraz University of Medical

Sciences, Shiraz, Iran

${ }^{6}$ Ionizing and Non-ionizing

Radiation Protection Re-

search Center (INIRPRC),

Shiraz University of Medical

Sciences, Shiraz, Iran

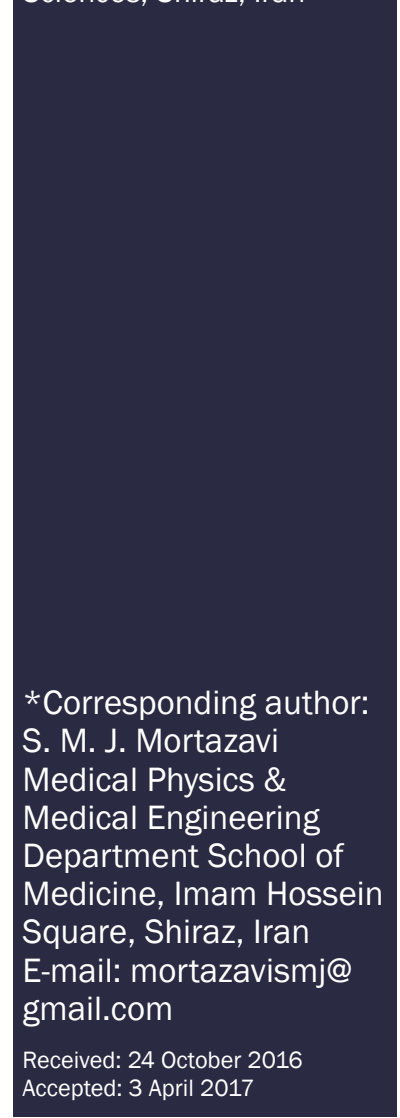

Accepted: 3 April 2017 
or difficulty in sharing their thoughts. Today, due to rapid changes in our modern world, generation, transmission and widespread applications of electricity have become essential parts of human life. Telecommunication technology has significantly improved communication, and nowadays, wireless systems can be found everywhere. It is widely believed that rapid advances in telecommunication infrastructure has led to the rise of global village that is playing a key role in decreasing the distance among people around the globe. Scientists believe that the past 10 years have been the most exciting era in terms of technological achievements in telecommunication. Therefore, now, all humans including pregnant women are continuously exposed to different levels of electromagnetic fields (EMFs) from a wide variety of sources such as mobile phones, mobile base stations and Wi-Fi.

Some studies show that exposure to electromagnetic radiation in the radiofrequency $(\mathrm{RF})$ range (RF-EMF) emitted by common mobile phones during pregnancy can be associated with adverse effects on the brain development in offspring which leads to hyperactivity. The occurrence of behavioral problems which mimic ADHD in laboratory animals have been shown to be caused by intrauterine exposure to RF-EMF of mobile phones [1]. Over the past several years, Mortazavi et al. have expanded their focus on studying the adverse health effects of exposure to different levels of radiofrequency electromagnetic fields (RFEMFs) such as cellular phones [2-13], mobile base stations $[14,15]$, Wi-Fi routers $[4,7,16$ 20]. The purpose of this study was to investigate whether maternal exposure to common sources of electromagnetic fields before and during pregnancy affect the rate and severity of speech problems in their offspring

\section{Materials and Methods}

\section{Ethical Issues}

This study was approved by the Medical
Ethics Committee of Shiraz University of Medical Sciences. All patients participating in this study were given informed consent prior to inclusion in the study.

\section{Design and samples}

This study has a cross-sectional design using a sample of 75 mothers of healthy children (control group) and 110 mothers of children aged 3-7 year diagnosed with speech problems who had been referred to a speech treatment center in Shiraz, Iran. Data were collected by interviews performed from June till December 2016 in Shiraz, Iran. Convenience sampling was used in this study, due to very limited availability of samples.

\section{Interview}

Following explanation of the goals of the study and its procedures and obtaining the participants' written consent, interviews were conducted with each mother. The participants were asked to verify whether they had been exposed to different sources of electromagnetic fields (e.g. mobile phones, cordless phones, CRTs, and high tension powerlines). A semistructured questionnaire was designed to assess all possible exposures to electromagnetic fields used for information recording.

\section{Data Analysis}

Data were analyzed using SPSS (Ver 19.0). A P-value of less than 0.05 was considered significant.

\section{Results}

Demographic and exposure data for study participants are summarized in Tables 1 and 2 . Among children with speech problems whose mothers were interviewed, $42.7 \%$ were female and $57.3 \%$ were male. This proportion for control children was $49.3 \%$ female and $50.7 \%$ male. The proportion of consanguineous marriage in the cases (mothers of children with speech problem) was $56.4 \%$, while in the control group it was only $37.3 \%$. All mothers 
Table 1: Demographic data of the mothers participated in this study.

\section{Demographic Data}

\begin{tabular}{lcc}
\hline \multicolumn{1}{l}{ Children's Sex } & & \\
\hline & Cases & $42.7 \%$ female, $57.3 \%$ male \\
\hline Marriage type & Control Group & $49.3 \%$ female, $50.7 \%$ male \\
\hline & Control Group & \\
\hline History of spontaneous abortion & $56.4 \%$ Consanguineous, $33.6 \%$ Non-consanguineous \\
\hline Cases & $37.3 \%$ Consanguineous, $62.7 \%$ Non-consanguineous \\
\hline Control Group & $20.6 \%$ Positive \\
\hline Other Children with speech problem & $17.4 \%$ Positive \\
\hline Cases & $14.3 \%$ Positive \\
\hline Control Group & $0 \%$ Positive
\end{tabular}

in both groups were non-smokers. Regarding exposure to ionizing radiation, only $2.8 \%$ of the cases and $2.7 \%$ of the controls had the history of dental radiography during pregnancy. In case of non-dental radiography, $2.4 \%$ of cases and $4 \%$ of the control group had the history of exposures during pregnancy.

For exposures to non-ionizing radiation, $97.6 \%$ of the cases and $100 \%$ of the controls had the history of mobile phone use before pregnancy. Moreover, $30.6 \%$ of the cases and $52.7 \%$ of the controls had the history of cordless phone use before pregnancy. The proportion of positive history of cathode ray tube (CRT) use before pregnancy in cases and controls were $24.1 \%$ and $13.3 \%$, respectively. History of exposure to high tension power lines during pregnancy was positive in $24.1 \%$ of the cases and $13.3 \%$ of the control group.

For exposures during pregnancy, $95.8 \%$ of the cases and $100 \%$ of the controls had the history of mobile phone use. Moreover, $30.6 \%$ of the cases and $50.7 \%$ of the controls had the history of cordless phone use pregnancy. The proportion of positive history of cathode ray tube (CRT) use pregnancy in cases and controls were $15.1 \%$ and $8.1 \%$, respectively. History of exposure to high tension power lines during pregnancy was positive in $15.4 \%$ of the cases and $1.4 \%$ of the control group.

Statistically significant associations were found between the use of cordless phone and offspring speech problems for both before pregnancy and during pregnancy maternal exposures $(\mathrm{P}=0.005$ and $\mathrm{P}=0.014$, respectively). However, cordless phone use before pregnancy was negatively linked to speech problems in offspring. Furthermore, significant associations were observed between the living in the vicinity of power lines and speech problems again for both before pregnancy and during pregnancy maternal exposures $(\mathrm{P}=0.003$ and $\mathrm{P}=0.002$, respectively). However, exposure to other sources of non-ionizing radiation including radiofrequency radiation emitted by mobile phones was not linked to speech problems. Moreover, exposure to ionizing radiation (e.g. radiography before and during pregnancy) was not associated with the occurrence of speech problems. 
Table 2: Exposure data of the mothers participated in this study.

Exposure Data

Significance

History of non-dental radiography during pregnancy

$\begin{array}{lll}\text { Cases } & 2.4 \% \text { Positive } & \text { NS } \\ \text { Control Group } & 4.0 \% \text { Positive }\end{array}$

History of dental radiography during pregnancy

$\begin{array}{cl}\text { Cases } & 2.8 \% \text { Positive } \\ \text { Control Group } & 2.7 \% \text { Positive }\end{array}$

History of non-dental radiography before pregnancy

$\begin{array}{lll}\text { Cases } & 34.8 \% \text { Positive } & \text { NS } \\ \text { Control Group } & 37.3 \% \text { Positive }\end{array}$

History of mobile phone use before pregnancy

\begin{tabular}{clll}
\hline Cases & $97.6 \%$ Positive & NS \\
\hline Control Group & $100 \%$ Positive & \\
\hline History of cordless phone use before pregnancy & & \\
\hline Cases & $30.6 \%$ Positive & P $=0.005$ \\
\hline Control Group & $52.7 \%$ Positive & \\
\hline
\end{tabular}

History of cathode ray tube (CRT) use before pregnancy

\begin{tabular}{lcl} 
Cases & $24.1 \%$ Positive & NS \\
Control Group & $13.3 \%$ Positive & \\
\hline Cases & & \\
Control Group & $10.9 \%$ Positive & $\mathrm{P}=0.003$ \\
\hline
\end{tabular}

History of mobile phone use during pregnancy

\begin{tabular}{clll}
\hline Cases & $95.8 \%$ Positive & NS \\
\hline Control Group & $100 \%$ Positive & \\
\hline History of cordless phone use during pregnancy & & \\
\hline Cases & $30.6 \%$ Positive & P $=0.014$ \\
\hline Control Group & $50.7 \%$ Positive & \\
\hline
\end{tabular}

History of cathode ray tube (CRT) use during pregnancy

$\begin{array}{ccc}\text { Cases } & 15.1 \% \text { Positive } & \text { NS } \\ \text { Control Group } & 8.1 \% \text { Positive }\end{array}$

History of exposure to high tension power lines during pregnancy

\begin{tabular}{ccc}
\hline & Cases & $15.4 \%$ Positive
\end{tabular}$\quad \mathrm{P}=0.002$




\section{Discussion}

The effect of electromagnetic fields on human neurons has been studied for decades [21]. While some studies could not show any neurobiological adverse effects in healthy and vulnerable RF EMF-exposed animals [22], other studies confirm the role of these exposures on neurodevelopment. We have previously reported the findings of our case-control study of children with clinically diagnosed speech problems and the controls. Our study showed a higher prenatal exposure to RFEMFs emitted from cell phones in speech problem group compared to controls [4]. As not only exposures before pregnancy were ignored in our previous study, all potential confounders were not adjusted in that study, we tried to improve the accuracy of data collection before and during pregnancy as well as to control for confounding factors. As far as we know, this is the first study to investigate a potential link between mothers' exposure to RF-EMFs both before and after pregnancy and offspring speech problems. It is worth noting that we have previously investigated whether maternal exposure to different sources of electromagnetic fields during pregnancy affect the rate and severity of speech problems in their offspring. Our previous study that was conducted on mothers of 35 healthy 3-5-yearold children (control group) and 77 children diagnosed with speech problems showed a significant association between either call time $(\mathrm{P}=0.002)$ or history of mobile phone use (months used) and speech problems in the offspring $(\mathrm{P}=0.003)$. In our previous study, other exposures had no effect on the occurrence of speech problems. As mentioned before, a major limitation of our previous study was ignoring the possible role of before pregnancy RFEMF exposures.

The findings of our new study failed to show a statistically significant association between the use of mobile phone and speech problems in the offspring either for maternal exposures before pregnancy or during pregnancy. How- ever, this study showed statistically significant associations between the use of cordless phone and speech problems in the offspring for both before pregnancy and during pregnancy maternal exposures $(\mathrm{P}=0.005$ and $\mathrm{P}=0.014$, respectively). Furthermore, significant associations were observed between the living in the vicinity of power lines and speech problems again for both before pregnancy and during pregnancy maternal exposures $(\mathrm{P}=0.003$ and $\mathrm{P}=0.002$, respectively). However, exposure to other sources of non-ionizing radiation including EMFs generated by CRT had no effect on the occurrence of speech problems. This was possibly due to high rate of mobile phone use in both groups. Given this consideration, as mobile phone use rates before pregnancy in cases and controls were 97.6 and $100 \%$, respectively and during pregnancy the rates were 95.8 and $100 \%$ in cases and controls, respectively, this study could not show any link between mobile phone use and speech problems in offspring. Our findings also showed that exposure to ionizing radiation (e.g. radiography before and during pregnancy) was not associated with the occurrence of speech problems.

Although this study has some limitations such as its small sample size and reliance on self-reporting, its findings lead us to this conclusion that the higher-than-ever levels of maternal exposure to some sources of RF-EMFs such as cordless phones or extremely low frequency electromagnetic fields (ELF-EMF) such as power lines can be associated with the occurrence of speech problems in the offspring. From a broader point of view, the findings obtained in this study cannot support the results reported by Taylor et al. who showed that maternal exposure to mobile phones during pregnancy can cause behavioral problems in mice which are similar to ADHD [1, 23]. Moreover, our new study cannot support the findings obtained in our previous study which showed a significant association between either the call time or history of mobile phone use (months used) and speech problems in the 
offspring [4]. In spite of this, our findings at least to some extent, are in line with a recent report which found no association between maternal cell phone use and low communication skills. Interestingly, this study even showed a beneficial association between maternal cell phone use during pregnancy and the child's neurodevelopment [24]. In our study, in a similar pattern, no cell phone use before pregnancy and during pregnancy were reported by $100 \%$ of control women, while no cell phone use before and during pregnancy in cases were $97.6 \%$ and $95.8 \%$, respectively.

Although this study has some limitations such as small sample size and reliance on selfreporting, it leads us to this conclusion that higher-than-ever levels of maternal exposure to some sources of ELF-EMF and RF-EMF could be linked to the offspring speech problems.

\section{Conclusion}

This study showed statistically significant associations between the use of cordless phone and offspring speech problems for both "before pregnancy" and "during pregnancy" maternal exposures. Regarding mobile phone use, due to high rate of mobile phone use in both groups, no association was found between mobile phone use and speech problems in offspring. Significant associations were observed between the living in the vicinity of power lines and speech problems again for "before pregnancy" and "during pregnancy" maternal exposures.

This study has some limitations but it leads us to this conclusion that higher-than-ever levels of maternal exposure to electromagnetic fields could be linked to the offspring speech problems.

\section{Acknowledgment}

This study was supported by the Ionizing and Non-ionizing Radiation Protection Research Center (INIRPRC), Shiraz University of Medical Sciences (SUMS), Shiraz, Iran.

\section{Footnotes}

S Zarei, M Vahab and MM Oryadi-Zanjani have equal contribution to this paper.

\section{Conflict of Interest}

None Declared

\section{References}

1. Peart KN [Internet]. Cell phone use in pregnancy may cause behavioral disorders in offspring. [cited 15 March 2012]. Available from: https://news.yale.edu/2012/03/15/cellphone-use-pregnancy-may-cause-behavioraldisorders-offspring

2. Mortazavi SM, Rahimi S, Talebi A, Soleimani A, Rafati A. Survey of the Effects of Exposure to $900 \mathrm{MHz}$ Radiofrequency Radiation Emitted by a GSM Mobile Phone on the Pattern of Muscle Contractions in an Animal Model. $J$ Biomed Phys Eng. 2015;5(3):121-32.

3. Mortazavi SAR, Mortazavi G, Mortazavi SMJ. Comments on "Radiofrequency electromagnetic fields and some cancers of unknown etiology: An ecological study". Sci Total Environ. 2017;609:1. doi: 10.1016/j.scitotenv.2017.07.131. PubMed PMID: 28732291.

4. Zarei S, Mortazavi SM, Mehdizadeh AR, Jalalipour M, Borzou S, Taeb S, et al. A Challenging Issue in the Etiology of Speech Problems: The Effect of Maternal Exposure to Electromagnetic Fields on Speech Problems in the Offspring. J Biomed Phys Eng. 2015;5:151-4. PubMed PMID: 26396971; PubMed Central PMCID: PMC4576876.

5. Mokarram P, Sheikhi M, Mortazavi SMJ, Saeb S, Shokrpour N. Effect of Exposure to 900 $\mathrm{MHz}$ GSM Mobile Phone Radiofrequency Radiation on Estrogen Receptor Methylation Status in Colon Cells of Male Sprague Dawley Rats. J Biomed Phys Eng. 2017;7:79-86. PubMed PMID: 28451581; PubMed Central PMCID: PMC5401136.

6. Eghlidospour M, Ghanbari A, Mortazavi SMJ, Azari $\mathrm{H}$. Effects of radiofrequency exposure emitted from a GSM mobile phone on proliferation, differentiation, and apoptosis of neural stem cells. Anat Cell Biol. 2017;50:11523. doi: 10.5115/acb.2017.50.2.115. PubMed 
PMID: 28713615; PubMed Central PMCID: PMC5509895.

7. Taheri M, Mortazavi SM, Moradi M, Mansouri S, Hatam GR, Nouri F. Evaluation of the Effect of Radiofrequency Radiation Emitted From Wi-Fi Router and Mobile Phone Simulator on the Antibacterial Susceptibility of Pathogenic Bacteria Listeria monocytogenes and Escherichia coli. Dose Response. 2017;15:1559325816688527. doi: 10.1177/1559325816688527. PubMed PMID: 28203122; PubMed Central PMCID: PMC5298474.

8. Mortazavi SAR, Mortazavi SMJ, Paknahad $M$. The role of electromagnetic fields in neurological disorders. J Chem Neuroanat. 2016;77:78-9. doi: 10.1016/j.jchemneu.2016.04.004. PubMed PMID: 27126876.

9. Mortazavi SM, Rouintan MS, Taeb S, Dehghan N, Ghaffarpanah AA, Sadeghi Z, et al. Human short-term exposure to electromagnetic fields emitted by mobile phones decreases computer-assisted visual reaction time. Acta Neurol Belg. 2012;112:171-5. doi: 10.1007/s13760012-0044-y. PubMed PMID: 22426673.

10.Mortazavi SM. Subjective Symptoms Related to GSM Radiation from Mobile Phone Base Stations: a cross-sectional study. J Biomed Phys Eng. 2014;4:39-40. PubMed PMID: 25505767; PubMed Central PMCID: PMC4258853.

11.Mortazavi SM, Motamedifar M, Namdari G, Taheri M, Mortazavi AR, Shokrpour N. Non-linear adaptive phenomena which decrease the risk of infection after pre-exposure to radiofrequency radiation. Dose Response. 2014;12:233-45. doi: 10.2203/ dose-response.12-055.Mortazavi. PubMed PMID: 24910582; PubMed Central PMCID: PMC4036396.

12.Mortazavi SM, Mahbudi A, Atefi M, Bagheri $\mathrm{S}$, Bahaedini N, Besharati A. An old issue and a new look: electromagnetic hypersensitivity caused by radiations emitted by GSM mobile phones. Technol Health Care. 2011;19:43543. doi: 10.3233/THC-2011-0641. PubMed PMID: 22129944.

13.Mortazavi SM, Ahmadi J, Shariati M. Preva- lence of subjective poor health symptoms associated with exposure to electromagnetic fields among university students. Bioelectromagnetics. 2007;28:326-30. doi: 10.1002/ bem.20305. PubMed PMID: 17330851.

14.Mortazavi S. Safety issues of mobile phone base stations. Journal of Biomedical Physics and Engineering. 2013;3.

15.Parsaei H, Faraz M, Mortazavi S. A multilayer perceptron neural network-based model for predicting subjective health symptoms in people living in the vicinity of mobile phone base stations. Ecopsychology. 2017;9:99105 doi: 10.1089/eco.2017.0011.

16. Mortazavi G, Mortazavi SM. Increased mercury release from dental amalgam restorations after exposure to electromagnetic fields as a potential hazard for hypersensitive people and pregnant women. Rev Environ Health. 2015;30:287-92. doi: 10.1515/ reveh-2015-0017. PubMed PMID: 26544100.

17.Mortazavi SA, Taeb S, Mortazavi SM, Zarei S, Haghani M, Habibzadeh P, et al. The Fundamental Reasons Why Laptop Computers should not be Used on Your Lap. J Biomed Phys Eng. 2016;6:279-84. PubMed PMID: 28144597; PubMed Central PMCID: PMC5219578.

18.Paknahad M, Mortazavi SM, Shahidi S, Mortazavi G, Haghani M. Effect of radiofrequency radiation from Wi-Fi devices on mercury release from amalgam restorations. J Environ Health Sci Eng. 2016;14:12. doi: 10.1186/s40201-016-0253-z. PubMed PMID: 27418965; PubMed Central PMCID: PMC4944481.

19.Shekoohi-Shooli F, Mortazavi SM, ShojaeiFard MB, Nematollahi S, Tayebi M. Evaluation of the Protective Role of Vitamin $C$ on the Metabolic and Enzymatic Activities of the Liver in the Male Rats After Exposure to 2.45 $\mathrm{GHz}$ Of Wi-Fi Routers. J Biomed Phys Eng. 2016;6:157-64. PubMed PMID: 27853723; PubMed Central PMCID: PMC5106548.

20.Taheri M, Mortazavi SM, Moradi M, Mansouri $S$, Nouri F, Mortazavi SA, et al. Klebsiella pneumonia, a Microorganism that Approves the Non-linear Responses to Antibiotics and 
Window Theory after Exposure to Wi-Fi 2.4 $\mathrm{GHz}$ Electromagnetic Radiofrequency Radiation. J Biomed Phys Eng. 2015;5:115-20. PubMed PMID: 26396967; PubMed Central PMCID: PMC4576872.

21.Perez F, Millholland G, Peddinti SV, Thella AK, Rizkalla J, Salama P, et al. Electromagnetic and Thermal Simulations of Human Neurons for SAR Applications. J Biomed Sci Eng. 2016;9:437-44. doi: 10.4236/ jbise.2016.99039. PubMed PMID: 27617054; PubMed Central PMCID: PMC5014390.

22.Petitdant N, Lecomte A, Robidel F, Gamez C, Blazy K, Villegier AS. Cerebral radiofrequency exposures during adolescence: Impact on astrocytes and brain functions in healthy and pathologic rat models. Bioelectromagnetics.
2016;37:338-50. doi: 10.1002/bem.21986. PubMed PMID: 27272062.

23.Aldad TS, Gan G, Gao XB, Taylor HS. Fetal radiofrequency radiation exposure from 800 1900 mhz-rated cellular telephones affects neurodevelopment and behavior in mice. Sci Rep. 2012;2:312. doi: 10.1038/srep00312. PubMed PMID: 22428084; PubMed Central PMCID: PMC3306017.

24.Papadopoulou E, Haugen M, Schjølberg S, Magnus P, Brunborg G, Vrijheid M, et al. Maternal cell phone use in early pregnancy and child's language, communication and motor skills at 3 and 5 years: the Norwegian mother and child cohort study (MoBa). BMC Public Health. 2017;17:685. doi: 10.1186/s12889017-4672-2. 